This is an Accepted Manuscript of an article published by Taylor \& Francis in International Journal of Control on 28/7/2016 available online: http://www.tandfonline.com/10.1080/00207179.2016.1207100 http:// authorservices.taylorandfrancis.com/sharing-your-work/ (Publisher journal website 9/11/2016)

\title{
Rigid Formation Control of Double-integrator Systems
}

\author{
Zhiyong Sun ${ }^{\mathrm{a} *}$, Brian D. O. Anderson ${ }^{\mathrm{a}}$, Mohammad Deghat ${ }^{\mathrm{b}}$, and Hyo-Sung Ahn ${ }^{\mathrm{c}}$ \\ ${ }^{a}$ Research School of Engineering, The Australian National University, Canberra, Australia; ${ }^{b}$ School of \\ Engineering and Information Technology, University of New South Wales at Canberra, Canberra, \\ Australia; ' School of Mechatronics, Gwangju Institute of Science and Technology, Gwangju, Republic of \\ Korea
}

(Received 00 Month 20XX; accepted 00 Month 20XX)

\begin{abstract}
In this paper we study rigid formation control systems modelled by double integrators. Two kinds of double-integrator formation systems are considered, namely, formation stabilization systems and flocking control systems. Novel observations on the measurement requirement, the null space and eigenvalues of the system Jacobian matrix will be provided, which reveal important properties of system dynamics and the associated convergence results. We also establish some new links between single-integrator formation systems and double-integrator formation systems via a parameterized Hamiltonian system, which in addition provide novel stability criteria for different equilibria in double-integrator formation systems by using available results in single-integrator formation systems.
\end{abstract}

Keywords: Rigid formation; double-integrator model; parameterized Hamiltonian system; equilibrium analysis

\section{Introduction}

Rigid formation shape control for a collection of $n$ point agents in Euclidean space is concerned with designing distributed control laws for individual agents so that the formation can converge to a prescribed rigid shape specified by a certain set of desired inter-agent distances Krick, Broucke, and Francis (2009), Oh, Park, and Ahn (2015). In the literature, most results on rigid formation control are based on simple single-integrator formation models; see e.g. Dimarogonas and Johansson (2008), Krick et al. (2009), Dörfler and Francis (2010), Helmke and Anderson (2013), Tian and Wang (2013), Anderson and Helmke (2014), Rozenheck, Zhao, and Zelazo (2015). In this paper, we will consider formation control systems modelled by double integrators, motivated by the fact that double-integrator systems serve as a somewhat more natural models to describe many real-life control systems.

Double-integrator models have been studied extensively for flocking control of multi-agent systems, partly originated by the pioneering work Olfati-Saber (2006) and Tanner, Jadbabaie, and Pappas (2007). Another active research topic on double-integrator models which received particular interest in recent years is the linear consensus problem Ren (2008), Cao, Yu, Ren, and Chen (2013). However, for rigid formation control systems modelled by double integrators, the results appear rather sparsely in the literature, with some discussions in Dimarogonas and Johansson (2008), Oh and Ahn (2014), Sun, Mou, Deghat, and Anderson (2015b), Cai and De Queiroz (2014); Ramazani, Selmic, and de Queiroz (2015); Zhang, de Queiroz, and Cai (2015). A recent paper Deghat, Anderson, and Lin (2015) showed the possibility of combining a linear consensus algorithm and a

*Corresponding author. Email: zhiyong.sun@anu.edu.au 
nonlinear rigid shape control to achieve a desired rigid flocking movement. Note that all these papers only focused on some local convergence analysis. Due to the nonlinear property of the formation controller for stabilizing rigid shapes, a full characterization of the convergence analysis is quite challenging. Actually, there are many open issues for rigid formation control systems when they are modelled by double integrators, which include equilibrium properties, convergence analysis, robustness issues, etc.

This paper provides new results dealing with the system dynamics and convergence property of double-integrator rigid formation systems. We will consider two types of formation systems: formation stabilization systems and flocking control systems. The main contributions of this paper are summarized as follows. First, we will characterize null spaces and zero eigenvalues of the Jacobian matrix for the vector function of the double-integrator formation system, which will reveal several system dynamical properties. Second, compared with the analysis and results in Dimarogonas and Johansson (2008), Oh and Ahn (2014), Sun et al. (2015b), Cai and De Queiroz (2014); Ramazani et al. (2015); Zhang et al. (2015) and Deghat et al. (2015), we go well beyond the local convergence analysis of the correct equilibrium set (i.e. those equilibria corresponding to correct formation shapes). Instead, we aim to provide new characterizations for the convergence properties of any equilibrium set, including those that do not correspond to the desired equilibrium. Third, invariant properties and links between single-integrator formation systems and doubleintegrator systems will be established. This will be done by employing a parameterized Hamiltonian system, an idea which was also used for power network analysis Chiang and Wu (1988), Chiang and Chu (1995), oscillator networks Dörfler and Bullo (2011), and was briefly mentioned in a recent paper Oh and Ahn (2014) on formation control. We will show how available results on characterizing equilibrium properties in single-integrator rigid formation systems (e.g. Dörfler and Francis (2010); Krick et al. (2009); Park, Sun, Anderson, and Ahn (2014); Sun, Helmke, and Anderson (2015a)) can be readily extended to the stability analysis for double-integrator formation systems. In our opinion, this is the most revealing of any method for analyzing the stability of double-integrator systems, since it ties their properties so closely to those of the more easily understood single-integrator rigid formation systems

This paper builds on a preliminary version Sun and Anderson (2015). The extensions presented in this paper include complete proofs for all the key results which were omitted in Sun and Anderson (2015), observations on measurement requirements and global/local coordinate frame issues, detailed discussions on certain properties of system dynamics, and a new section with supportive simulations. We mention that this paper does not focus on proposing novel controllers for stabilizing rigid formation shapes. Instead, we study double-integrator rigid formation systems with the popular formation stabilization controller proposed in Krick et al. (2009), while the main purposes of this paper are to provide more understanding and insights on the system dynamics and convergence analysis which will help the implementation of such formation controllers in practice.

The remaining parts of this paper are organized as follows. Section 2 briefly reviews some background and then introduces the relevant system equations. Section 3 presents results on local convergence and Jacobian matrix analysis using linearization technique. By exploring a parameterized Hamiltonian system, Section 4 establishes certain invariance principles, which help to characterize novel equilibrium properties on double-integrator formation systems by relating them to available results in single-integrator formation systems. Extensions of the results to flocking formation systems are discussed in Section 5. Some illustrative examples with simulation results are shown in Section 6. Finally, conclusions are provided in Section 7 which closes this paper.

Notations. The notations used in this paper are fairly standard. The rank, determinant and null space of a matrix $M$ are denoted by $\operatorname{rank}(M), \operatorname{det}(M)$ and null $(M)$, respectively. We use $\operatorname{span}\left\{v_{1}, v_{2}, \cdots, v_{k}\right\}$ to represent the subspace spanned by a set of $k$ vectors $v_{1}, v_{2}, \cdots, v_{k}$. We denote the $n \times n$ identity matrix and zero matrix as $I_{n \times n}$ and $\mathbf{0}_{n \times n}$, respectively. Let $\mathbf{1}_{n}$ and $\mathbf{0}_{n}$ be the $n$-dimensional vectors of all ones and all zeros. When the subscripts for $I_{n \times n}, \mathbf{0}_{n \times n}, \mathbf{1}_{n}$ or $\mathbf{0}_{n}$ are omitted, their dimensions should be clear in the context. The inertia of a matrix $M \in \mathbb{R}^{n \times n}$ 
is given by the triple $\left\{\nu_{+}, \nu_{-}, \nu_{0}\right\}$, where $\nu_{+}$(respectively, $\nu_{-}$) denotes the number of unstable (respectively, stable) eigenvalues of $M$ in the open right (respectively, left) complex half plane, and $\nu_{0}$ denotes the number of eigenvalues with zero real part (see e.g. Carlson and Schneider (1963)). The symbol $\otimes$ denotes Kronecker product. $S E(d)$ and $S O(d)$ denote the special Euclidean group and the special orthogonal group, respectively.

\section{Background and system equations}

\subsection{Basic concepts on graph and rigidity theory}

Consider an undirected simple graph with $m$ edges and $n$ vertices, denoted by $\mathcal{G}=(\mathcal{V}, \mathcal{E})$ with vertex set $\mathcal{V}=\{1,2, \cdots, n\}$ and edge set $\mathcal{E} \subset \mathcal{V} \times \mathcal{V}$. The neighbor set $\mathcal{N}_{i}$ of node $i$ is defined as $\mathcal{N}_{i}:=\{j \in \mathcal{V}:(i, j) \in \mathcal{E}\}$. The matrix relating the nodes to the edges is called the incidence matrix $H=\left\{h_{k i}\right\} \in \mathbb{R}^{m \times n}$, whose entries are defined as (with arbitrary edge orientations for the undirected formation considered here)

$$
h_{k i}=\left\{\begin{array}{cc}
1, & \text { the } k \text {-th edge sinks at node } i \\
-1, & \text { the } k \text {-th edge leaves node } i \\
0, & \text { otherwise }
\end{array}\right.
$$

The Laplacian matrix $L(\mathcal{G})$ will often be used for matrix representation of a graph $\mathcal{G}$, which is defined as $L(\mathcal{G})=H^{T} H$ for undirected graphs. For a connected undirected graph, there holds $\operatorname{rank}(L)=n-1$ and $\operatorname{null}(L)=\operatorname{null}(H)=\operatorname{span}\left\{\mathbf{1}_{n}\right\}$.

Let $p_{i} \in \mathbb{R}^{d}$ where $d=\{2,3\}$ denotes a point that is assigned to $i \in \mathcal{V}$. The stacked vector $p=\left[p_{1}^{T}, p_{2}^{T}, \cdots, p_{n}^{T}\right]^{T} \in \mathbb{R}^{d n}$ represents the realization of $\mathcal{G}$ in $\mathbb{R}^{d}$. The pair $(\mathcal{G}, p)$ is said to be a framework (specifically, a formation in the context of our control problem) of $\mathcal{G}$ in $\mathbb{R}^{d}$. By introducing the matrix $\bar{H}:=H \otimes I_{d \times d} \in \mathbb{R}^{d m \times d n}$, one can construct the relative position vector $z$ as follows

$$
z=\bar{H} p
$$

where $z=\left[z_{1}^{T}, z_{2}^{T}, \cdots, z_{m}^{T}\right]^{T} \in \mathbb{R}^{d m}$, with $z_{k} \in \mathbb{R}^{d}$ being the relative position vector for the vertex pair defined by the $k$-th edge.

Define $Z(z)=\operatorname{diag}\left(z_{1}, z_{2}, \cdots, z_{m}\right) \in \mathbb{R}^{d m \times m}$. With this notation at hand, we consider the smooth distance map

$$
r_{\mathcal{G}}: \mathbb{R}^{d n} \longrightarrow \mathbb{R}^{m}, r_{\mathcal{G}}(p)=\left(\left\|p_{i}-p_{j}\right\|^{2}\right)_{(i, j) \in \mathcal{E}}=Z^{T} z .
$$

This paper focuses on formation control of rigid shapes. The definition of graph rigidity can be found in e.g. Hendrickson (1992). A useful tool to study graph rigidity is the rigidity matrix, which is defined as the Jacobian matrix $R(p)=\frac{1}{2} \partial r_{\mathcal{G}}(p) / \partial(p)$. By inspection, $R(p)$ is an $m \times d n$ matrix given as

$$
R(p)=Z(z)^{T} \bar{H}
$$

Note that the entries of $R(p)$ involve only relative position vectors $z$, and we can rewrite it as $R(z)$. The rank of rigidity matrix can be used to determine the infinitesimal rigidity of the framework, as shown in the following theorem. 
Theorem 1: (Hendrickson (1992)) Consider a framework $(\mathcal{G}, p)$ in d-dimensional space with $n \geq d$ vertices and $m$ edges. It is infinitesimally rigid if and only if

$$
\operatorname{rank}(R(p))=d n-d(d+1) / 2
$$

\subsection{Motion equations}

Let $d_{k_{i j}}$ denote the desired length of edge $k$ which links agent $i$ and $j$. We assume that the set of desired lengths is realizable, i.e., there exists a formation in $\mathbb{R}^{d}$ whose inter-agent distances correspond to the desired values. In the following, the set of all formations $(\mathcal{G}, p)$ which satisfies the distance constraints is referred to as the set of target formations. ${ }^{1}$ In this paper we assume that the target formation is infinitesimally rigid.

We further define (for an arbitrary formation)

$$
e_{k_{i j}}=\left\|p_{i}-p_{j}\right\|^{2}-d_{k_{i j}}^{2}=\left\|z_{k}\right\|^{2}-d_{k_{i j}}^{2}
$$

to denote the squared distance error for edge $k$. Note we may also use $e_{k}$ and $d_{k}$ occasionally for notational convenience in the sequel if no confusion is expected. The squared distance error vector is denoted by $e=\left[e_{1}, e_{2}, \cdots, e_{m}\right]^{T}$.

Most papers on rigid formation control have considered the following formation control system modelled by a single integrator

$$
\dot{p}_{i}=-\sum_{j \in \mathcal{N}_{i}}\left(\left\|p_{i}-p_{j}\right\|^{2}-d_{k_{i j}}^{2}\right)\left(p_{i}-p_{j}\right), \quad i=1, \ldots, n
$$

which defines the steepest descent gradient flow of the distance potential function

$$
V(p)=\frac{1}{4} \sum_{(i, j) \in \mathcal{E}}\left(\left\|p_{i}-p_{j}\right\|^{2}-d_{k_{i j}}^{2}\right)^{2}
$$

In a compact form, we can rewrite (6) as

$$
\dot{p}(t)=-\nabla_{p} V=-R^{T}(z) e(z)
$$

Two kinds of double-integrator rigid formation systems will be considered in this paper. The first one is a model with velocity damping term, which aims to stabilize a desired rigid shape and to achieve a stationary formation (i.e. the final formation should come to a rest). The formation system can be described by the following form:

$$
\begin{aligned}
& \dot{p}_{i}=v_{i} \\
& \dot{v}_{i}=-k_{i} v_{i}-\sum_{j \in \mathcal{N}_{i}}\left(\left\|p_{i}-p_{j}\right\|^{2}-d_{k_{i j}}^{2}\right)\left(p_{i}-p_{j}\right)
\end{aligned}
$$

where $v_{i} \in \mathbb{R}^{d}$ is the velocity of agent $i$ and $k_{i}$ is a positive control gain which is freely selectable by the system designer. Define

$$
\psi(p, v):=\frac{1}{2} \sum_{i \in \mathcal{V}}\left\|v_{i}\right\|^{2}+V(p)
$$

\footnotetext{
${ }^{1}$ The realization of a target formation with the given desired distances may not be unique up to rotation and translation Hendrickson (1992).
} 
Then in a compact form, we can rewrite the above double-integrator formation stabilization system as

$$
\begin{aligned}
\dot{p} & =\nabla_{v} \psi=v \\
\mathcal{H}_{\text {formation }}: \dot{v} & =-\mathcal{K} \nabla_{v} \psi-\nabla_{p} \psi \\
& =-\mathcal{K} v-R^{T}(z) e(z)
\end{aligned}
$$

where $\mathcal{K}=K \otimes I_{d \times d}$ and $K$ is a diagonal gain matrix with the $i$-th diagonal entry being $k_{i}$.

The other model is for achieving a flocking behavior with both velocity consensus and shape stabilization. The overall system can be described by the following equations ${ }^{2}$

$$
\begin{aligned}
& \dot{p}_{i}=v_{i} \\
& \dot{v}_{i}=\sum_{j \in \mathcal{N}_{i}}\left(v_{j}-v_{i}\right)-\sum_{j \in \mathcal{N}_{i}}\left(\left\|p_{i}-p_{j}\right\|^{2}-d_{k_{i j}}^{2}\right)\left(p_{i}-p_{j}\right)
\end{aligned}
$$

In a compact form, we can rewrite the above double-integrator flocking control system as

$$
\mathcal{H}_{\text {flocking }}: \begin{aligned}
& \dot{p}=\nabla_{v} \psi=v \\
& \dot{v}=-\mathcal{L} v-R^{T}(z) e(z)
\end{aligned}
$$

where $\mathcal{L}=L \otimes I_{d \times d}$ and $L$ is the Laplacian matrix for the underlying undirected and connected graph.

It is noted that different distance potential functions, other than the one in (7), can be chosen for stabilizing rigid formation shapes, which will give rise to different types of formation controllers. For the case of general potential functions, a unified approach is available for conducting convergence analysis (see relevant discussions in Sun, Mou, Anderson, and Cao (2016)). In this paper, we will focus on the potential function (7) to model double-integrator formation systems, while more general potential functions can also be used by following similar analysis in Sun et al. (2016).

\section{$2.3 \quad$ Independence of global coordinate frame}

We state the following lemma on the coordinate system requirement for implementing the formation control law (11) and the flocking control law (13).

Lemma 1: Suppose each agent can access its own velocity. Then the implementation of the doubleintegrator formation control system (11) does not require all agents' coordinate systems to be aligned. Furthermore, suppose each agent can measure the relative velocity to its neighbors in addition to its own velocity. Then the implementation of the double-integrator flocking system (13) does not require all agents' coordinate systems to be aligned.

Proof. The proof for the first statement in the lemma can be handled in the same way as the proof of the second statement, so we will focus on the proof of the second statement.

Suppose agent $i$ 's position and velocity in the global coordinate system $\sum_{g}$ are measured as $p_{i}^{g}$ and $v_{i}^{g}$, while $p_{i}^{i}, p_{j}^{i}, v_{i}^{i}$, and $v_{j}^{i}$ denote agent $i$ 's and its neighboring agent $j$ 's positions and velocities, respectively, measured by agent $i$ 's local coordinate system. The controller for agent $i$ is implemented in its local coordinate as

\footnotetext{
${ }^{2}$ Note that there are two types of velocity consensus algorithms depending on different underlying graphs: one is based on undirected underlying graph and the other is based on directed graph (for achieving a leader-following control). Furthermore, the underlying graph for achieving velocity consensus can be different to the one of shape stabilization (see relevant discussions in Deghat et al. (2015); Qin and Yu (2013)). In this paper we focus on the first one (with undirected underlying graph for the velocity consensus) and assume the same underlying graph for both shape stabilization and velocity consensus.
} 


$$
\ddot{p}_{i}^{i}=u_{i}^{i}=\sum_{j \in \mathcal{N}_{i}}\left(v_{j}^{i}-v_{i}^{i}\right)-\sum_{j \in \mathcal{N}_{i}}\left(\left\|p_{i}^{i}-p_{j}^{i}\right\|^{2}-d_{k_{i j}}^{2}\right)\left(p_{i}^{i}-p_{j}^{i}\right)
$$

Clearly, there exist a rotation matrix $\mathcal{Q}_{i} \in S O(d)$ and a translation vector $\vartheta_{i} \in \mathbb{R}^{d}$, such that $p_{j}^{i}=\mathcal{Q}_{i} p_{j}^{g}+\vartheta_{i}$ and $v_{j}^{i}-v_{i}^{i}=\mathcal{Q}_{i}\left(v_{j}^{g}-v_{i}^{g}\right)$. We rewrite the controller (14) for agent $i$ in the global coordinate system $\sum_{g}$ as follows

$$
\begin{aligned}
\ddot{p}_{i}^{g} & =\mathcal{Q}_{i}^{-1} u_{i}^{i}=\mathcal{Q}_{i}^{-1}\left(\sum_{j \in \mathcal{N}_{i}}\left(v_{j}^{i}-v_{i}^{i}\right)-\sum_{j \in \mathcal{N}_{i}}\left(\left\|p_{i}^{i}-p_{j}^{i}\right\|^{2}-d_{k_{i j}}^{2}\right)\left(p_{i}^{i}-p_{j}^{i}\right)\right) \\
& =\sum_{j \in \mathcal{N}_{i}}\left(v_{j}^{g}-v_{i}^{g}\right)-\sum_{j \in \mathcal{N}_{i}}\left(\left\|p_{i}^{g}-p_{j}^{g}\right\|^{2}-d_{k_{i j}}^{2}\right) \mathcal{Q}_{i}^{-1} \mathcal{Q}_{i}\left(p_{i}^{g}-p_{j}^{g}\right) \\
& =\sum_{j \in \mathcal{N}_{i}}\left(v_{j}^{g}-v_{i}^{g}\right)-\sum_{j \in \mathcal{N}_{i}}\left(\left\|p_{i}^{g}-p_{j}^{g}\right\|^{2}-d_{k_{i j}}^{2}\right)\left(p_{i}^{g}-p_{j}^{g}\right)
\end{aligned}
$$

which has the same form as (14). Since $\mathcal{Q}_{i}$ and $\vartheta_{i}$ are chosen arbitrarily, the above equation indicates that the designed controllers for all agents are independent of the global coordinate basis. The proof for the first statement can be handled by modifying $v_{j}^{i}-v_{i}^{i}$ as $v_{i}^{i}$ in the above equation, and similar argument then follows.

In the remaining analysis in later sections, in order to avoid complicated notation, we will drop the superscript in the controller term (15) and still use the expression of (9) or (12) for the convenience of analysis.

Remark 1: The above controller property, termed translational and rotational invariance property (or the $S E(d)$ invariance property Vasile, Schwager, and Belta (2015)), has been well studied for single-integrator rigid formation control systems (see e.g. Krick et al. (2009)). To the best of our knowledge, the corresponding property for double-integrator rigid formation systems has been largely ignored in the previous literature. Lemma 1 highlights the advantage of rigid formation controllers, in contrast to the displacement-based formation control strategy (see the survey Oh et al. (2015)). Note that in the latter case in which the formation shape is defined by a set of desired relative positions, all the agents need to align their local coordinate frames to be the same as that of the global coordinate frame. If there exists even a slight alignment mismatch, the displacementbased formation control may lead to undesired formation motions or even formation failure Meng, Anderson, and Hirche (2016).

Remark 2: In practical formation control one needs to distinguish different ways of obtaining the required relative information for each individual agent (e.g., either by measurement solely at one agent or measurement additionally at neighboring agents with communication). In the statement of Lemma 1 , we assume the relative velocity $v_{j}-v_{i}$ is measured using agent $i$ 's local coordinate system which is usually the case for implementing a formation control system in practice. If alternatively, the neighboring agents' velocities were transmitted to agent $i$, then adjacent agents would need to align their local coordinate systems or have the knowledge of the orientation of a common coordinate system so that the communicated velocity information can be correctly interpreted. 


\section{System dynamics}

\subsection{Equilibrium set for single- and double-integrator formation systems}

We first describe the equilibrium set of each formation system. Suppose the distance potential (7) and the target formation are the same for the single-integrator system (8) and double-integrator systems (11) (used in the composite potential (10)) and (13). Denote the set of all equilibria of the single-integrator system (8) as

$$
\mathcal{M}_{S}:=\left\{p^{*}: \nabla_{p} V\left(p^{*}\right)=\mathbf{0}\right\}
$$

First note that the distance potential $V$ defined in (7) for shape stabilization only depends on distances between agents and thus is invariant with respect to translation and rotation. Such symmetries imply that any formation system that involves the gradient of the distance potential $V$ in (7) does not have isolated equilibrium points. Instead, it possesses continuum equilibria which form orbits under the $S E(d)$ group action. To fix ideas, let us define the $S E(d)$ group action to a configuration $P=\left[p_{1}, p_{2}, \cdots, p_{n}\right]$ of $n$ agents' positions with each $p_{i} \in \mathbb{R}^{d}$ :

$$
\mathcal{O}_{P}:=S E(d) \cdot P=\left[\mathcal{Q} p_{1}+\vartheta, \mathcal{Q} p_{2}+\vartheta, \cdots, \mathcal{Q} p_{n}+\vartheta\right]
$$

where $\mathcal{Q} \in S O(d)$ and $\vartheta \in \mathbb{R}^{d}$ are components of $S E(d)$. We call $\mathcal{O}_{P}$ the orbit of $P$ under the group action of $S E(d)$. From this it is obvious to see that if $p^{*}$ is an equilibrium point of (8), then any point generated by the action $\mathcal{O}_{P^{*}}$ is also an equilibrium point. Similarly to the notion used in Helmke and Anderson (2013) and Chen, Belabbas, and Başar (2015), we will call $\mathcal{O}_{P^{*}}$ an equilibrium orbit. As a consequence, the Hessian for the gradient-based rigid formation system (8) is always singular, with parts of the null spaces induced by the shape invariance under rotation and translation. If the Hessian at an equilibrium point $p^{*}$ of (8) is nonsingular in the normal direction to $\mathcal{O}_{P^{*}}$, then we will say it is normal hyperbolic.

Similarly, for the corresponding double-integrator formation system (11), the set of all equilibria can be described as

$$
\mathcal{M}_{D}:=\left\{\left(p^{*}, v^{*}\right): \nabla_{p} V\left(p^{*}\right)=\mathbf{0}, v^{*}=\mathbf{0} \in \mathbb{R}^{d n}\right\}
$$

Finally, for the double-integrator flocking system (13), we define the set

$$
\mathcal{M}_{F}:=\left\{\left(p^{*}, v^{*}\right): \nabla_{p} V\left(p^{*}\right)=\mathbf{0}, \mathcal{L} v^{*}=\mathbf{0}\right\}=\left\{\left(p^{*}, v^{*}\right): \nabla_{p} V\left(p^{*}\right)=\mathbf{0}, v^{*}=\mathbf{1}_{n} \otimes v_{c}\right\}
$$

where $v_{c}$ is the average velocity. In the later analysis, we will call the set (19) defined above the set of equilibrium orbits for the double-integrator flocking system, according to the fact that all agents will finally show a steady equilibrium motion which is a flocking motion. The above discussions on continuum equilibria and equilibrium orbits under $S E(d)$ group action for the single-integrator system (8) also apply to the property of the equilibrium set defined above for the double-integrator formation stabilization system (11) and flocking system (13).

By the definition in (19), $v_{c}$ is the velocity of the formation centroid. The existence of a constant $v_{c}$, readily computable from the initial conditions, is shown by the following lemma.

Lemma 2: For the double-integrator flocking system (13), the velocity of the formation centroid $v_{c}$ remains fixed throughout the motion.

Proof. Denote by $p_{c} \in \mathbb{R}^{d}$ the center of the mass of the formation, i.e., $p_{c}=\frac{1}{n} \sum_{i=1}^{n} p_{i}=\frac{1}{n}\left(\mathbf{1}_{n} \otimes\right.$ 
$\left.I_{d}\right)^{T} p$ and thus

$$
v_{c}=\dot{p}_{c}=\frac{1}{n}\left(\mathbf{1}_{n} \otimes I_{d}\right)^{T} \dot{p}
$$

From (13), one can show

$$
\begin{aligned}
\dot{v}_{c} & =\frac{1}{n}\left(\mathbf{1}_{n} \otimes I_{d}\right)^{T} \ddot{p} \\
& =\frac{1}{n}\left(\mathbf{1}_{n} \otimes I_{d}\right)^{T}\left(-\mathcal{L} v-R^{T} e\right) \\
& =-\frac{1}{n}\left(\mathbf{1}_{n} \otimes I_{d}\right)^{T} \mathcal{L} v-\frac{1}{n}\left(Z^{T}\left(H \otimes I_{d}\right)\left(\mathbf{1}_{n} \otimes I_{d}\right)\right)^{T} e
\end{aligned}
$$

Note that $\left(\mathbf{1}_{n} \otimes I_{d}\right)^{T} \mathcal{L} v=\mathbf{0}$. Also because $\operatorname{null}(H)=\operatorname{span}\left\{\mathbf{1}_{n}\right\}$ there holds $\left(Z^{T}\left(H \otimes I_{d}\right)\left(\mathbf{1}_{n} \otimes I_{d}\right)\right)^{T} e=\mathbf{0}$. Thus $\dot{v}_{c}=\mathbf{0}$, which indicates that the velocity of the formation centroid remains constant.

Based on Lemma 2, a commonly-used approach for stability analysis of the flocking control system is to perform a linear system transformation by separating the motion of the formation centroid (see e.g., Deghat et al. (2015)). We will also discuss this point in Section 5.

In the following we introduce the notion of set stability (Hahn and Baartz, 1967, Chapter V) which will be used later. Define the distance between a point $p$ and a set $S$ as

$$
\operatorname{dist}(p, S)=\inf _{y \in S}\|p-y\|
$$

This point to set distance allows us to define the stability for a set, which can be seen as a straightforward extension of the well known definition of that for an equilibrium point. We omit the detailed definitions here, and refer the readers to (Hahn and Baartz, 1967, Chapter V) for definitions and explanations on concepts such as set stability, asymptotic set stability and exponential set stability. The slight difference between the set stability and a closely related concept termed orbital stability (see (Wiggins, 2003, Chapter 1.2) for detailed discussions) was discussed in (Hahn and Baartz, 1967, Page 171), and there will be no confusion caused by this slight difference in the context of formation control (since an equilibrium orbit under $S E(d)$ group action is also defined as an equilibrium set). In this paper, when no confusion is expected, we will use these two terms orbital stability and set stability interchangeably.

In general, rigid formation control systems exhibit multiple equilibrium orbits (see discussions in Anderson and Helmke (2014), Sun et al. (2015a)). We call the set of equilibria at which the correct shape is attained the correct equilibrium set (or correct equilibrium orbit), and we denote it by $\mathcal{M}_{S}^{c}:=\left\{p^{*}: e\left(p^{*}\right)=\mathbf{0}\right\}$ for the single-integrator system $(8), \mathcal{M}_{D}^{c}=\left\{\left(p^{*}, \mathbf{0}\right): e\left(p^{*}\right)=\mathbf{0}\right\}$ for the double-integrator formation system (11), and $\mathcal{M}_{F}^{c}=\left\{\left(p^{*}, v^{*}\right): e\left(p^{*}\right)=\mathbf{0}, v^{*}=\mathbf{1}_{n} \otimes v_{c}\right\}$ for the double-integrator flocking system (13). Note that a correct equilibrium set is characterized by the condition $e\left(p^{*}\right)=\mathbf{0}$, i.e., the set of desired distances at such an equilibrium is achieved. Correspondingly, one can also define incorrect equilibrium set by following the property that at such an equilibrium the set of desired distances is not achieved (i.e. $e\left(p^{*}\right) \neq \mathbf{0}$ ).

Remark 3: We also note that the set of correct equilibria (up to translation, rotation and reflection) may not form a unique equilibrium orbit. This is because the realization of a target formation with given distances (up to translation, rotation and reflection) may not be unique due to e.g., flex or flip ambiguity Hendrickson (1992). In other words, two correct equilibria may not correspond to congruent formations, since for minimally rigid formations with more than three agents, there 
always exist noncongruent equilibria pairs obtainable by either a flex or flip ambiguity Anderson, Yu, Fidan, and Hendrickx (2008).

\subsection{General results on convergence analysis}

The following convergence results for double-integrator formation systems (11) and (13) are well known, and are based on standard Lyapunov argument and LaSalle's Invariance Principle.

Lemma 3: Each trajectory of the double-integrator formation system (11) converges to an invariant set in $\mathcal{M}_{D}$. Also, each trajectory of the double-integrator flocking system (13) converges to an invariant set in $\mathcal{M}_{F}$.

The proof can be shown by following similar steps from e.g. Deghat et al. (2015), Sun et al. (2015b) and is omitted here.

The following result characterizes the local convergence of the correct equilibrium set.

Corollary 1: There exists a neighborhood of $\mathcal{M}_{D}^{c}$ such that the trajectory of (11) converges locally to an equilibrium point in $\mathcal{M}_{D}^{c}$. Also, there exists a neighborhood of $\mathcal{M}_{F}^{c}$ such that the trajectory of (13) converges locally to $\mathcal{M}_{F}^{c}$.

We remark that the first statement in Corollary 1 indeed shows a stronger convergence result, i.e., the convergence to a point in the equilibrium set (rather than the convergence to the set).

The local convergence to the correct equilibrium set for double-integrator formation systems as shown in Corollary 1 has been proved in several previous papers based on different assumptions on graph topologies and formation characterizations. For example, in Dimarogonas and Johansson (2008), the local convergence for double-integrator formation stabilization system (11) was proved by assuming that the underlying topology is a tree (and in this case the local convergence to a correct equilibrium set can be extended to global convergence). In Sun et al. (2015b), the local convergence for the double-integrator flocking system (13) was proved by assuming that the target formation is minimally and infinitesimally rigid, via Lyapunov arguments and LaSalle's Invariance Principle. A more recent paper Deghat et al. (2015) presented a comprehensive study for the double-integrator formation flocking system (13), by employing the linearization approach and the Center Manifold Theorem Carr (1981). Note that Deghat et al. (2015) only assumed that the target formation is infinitesimally rigid, which is a more relaxed assumption than that in Sun et al. (2015b). Also note that Deghat et al. (2015) mostly focuses on the stability analysis for the formation flocking system (13), while the analysis and proof can be directly extended to the case of formation stabilization system (11). Hence, we omit the proof for Corollary 1 here and refer the readers to the above references.

Note that the above results only concern the local convergence property of the correct equilibrium set. It is still an open problem in the field of rigid formation control to determine or rule out the existence of stable incorrect equilibria (cf. Anderson and Helmke (2014); Sun et al. (2015a)). In Section 4.2 we will prove the instability for a special set of incorrect equilibria called degenerate equilibria after we establish a link between single-integrator rigid formation systems and doubleintegrator rigid formation systems.

\subsection{Jacobian matrix analysis}

In this subsection we will show new results on system dynamics at a general equilibrium by analyzing the Jacobian matrix via the linearization technique. Note that from this subsection we will focus on the double-integrator formation control system (11) for the convenience of analysis, but note that most results can be extended to the corresponding flocking model (13) with slight 
modifications. In Section 5, we will show how to extend the results from the formation control system (11) to the flocking control system (13).

Denote the Jacobian matrix of the right-hand vector function of (8) at an equilibrium point $p^{*}$ as

$$
\begin{aligned}
J_{p^{*}} & =\left.\frac{\partial\left(-R^{T}(p) e(p)\right)}{\partial p}\right|_{p=p^{*}} \\
& =\left.\left(-\frac{\partial R^{T}(p)}{\partial p} e(p)-R^{T}(p) \frac{\partial e(p)}{\partial p}\right)\right|_{p=p^{*}}
\end{aligned}
$$

Note that because (8) describes a gradient descent flow of $V(p), J_{p^{*}}$ is actually the Hessian matrix of $-V(p)$ and thus is symmetric. Define a diagonal matrix $E=\operatorname{diag}\left(e_{1}, e_{2}, \cdots, e_{m}\right) \in \mathbb{R}^{m \times m}$. Then an explicit expression of $J_{p^{*}}$ can be obtained as (see e.g. Anderson and Helmke (2014), Sun et al. (2015a))

$$
\begin{aligned}
J_{p^{*}} & =-2 R\left(p^{*}\right)^{T} R\left(p^{*}\right)-\left(H^{T} E\left(p^{*}\right) H\right) \otimes I_{d} \\
& =\bar{H}^{T}\left(-2 Z\left(p^{*}\right) Z\left(p^{*}\right)^{T}-E\left(p^{*}\right) \otimes I_{d}\right) \bar{H}
\end{aligned}
$$

Note that, as can be seen from the above formula (23), the null space of $J_{p^{*}}$ is always non-empty. For example, in the 2-D case, the dimension of null $\left(J_{p^{*}}\right)$ is at least three, with two dimensions reflecting the translation invariance and one dimension reflecting the rotation invariance of a rigid shape. In the general case, at a correct equilibrium at which $E\left(p^{*}\right)$ is a zero matrix, there holds $J_{p^{*}}=-2 R\left(p^{*}\right)^{T} R\left(p^{*}\right)$ and the dimension of the null space of $J_{p^{*}}$ is $d(d+1) / 2$ according to Theorem 1.

The Jacobian matrix of the double-integrator formation system (11) at an equilibrium point $\left(p^{*}, \mathbf{0}\right)$ can be calculated as

$$
J\left(p^{*}, \mathbf{0}\right)=\left[\begin{array}{cc}
\mathbf{0}_{d n \times d n} & I_{d n \times d n} \\
J_{p^{*}} & -\mathcal{K}
\end{array}\right]
$$

where $J_{p^{*}}$ is the Jacobian matrix of (8) defined in (22), and $\mathcal{K}$ is the diagonal gain matrix defined in (11).

The following result characterizes the null space of $J\left(p^{*}, \mathbf{0}\right)$.

Theorem 2: Suppose the null space of $J_{p^{*}}$ is spanned by a set of l linearly independent vectors $\nu_{j}$ and denote the null space by $\operatorname{null}\left(J_{p^{*}}\right)=\operatorname{span}\left\{\nu_{1}, \cdots, \nu_{j}, \cdots, \nu_{l}\right\}$. Define the corresponding vector $v_{j}=\left[\nu_{j}^{T}, \mathbf{0}^{T}\right]^{T}$. Then there holds null $\left(J\left(p^{*}, \mathbf{0}\right)\right)=\operatorname{span}\left\{v_{1}, \cdots, v_{j}, \cdots, v_{l}\right\}$.

Proof. Suppose $v_{j}=\left[v_{j p}^{T}, v_{j v}^{T}\right]^{T}$ is a vector in the null space of $J\left(p^{*}, \mathbf{0}\right)$, that is

$$
\left[\begin{array}{cc}
\mathbf{0}_{d n \times d n} & I_{d n \times d n} \\
J_{p^{*}} & -\mathcal{K}
\end{array}\right]\left[\begin{array}{c}
v_{j p} \\
v_{j v}
\end{array}\right]=0
$$

which can be simplified as

$$
\begin{aligned}
v_{j v} & =\mathbf{0} \\
J_{p^{*}} v_{j p}-\mathcal{K} v_{j v} & =\mathbf{0}
\end{aligned}
$$

The above equation implies that $v_{j p}$ is the corresponding vector in the null space of $J_{p^{*}}$. Thus, there holds $\nu_{j}:=v_{j p}$. Also note that each vector $\nu_{j}$ in the null space of $J_{p^{*}}$ gives rise to one vector 
$v_{j}$ in the null space of $J\left(p^{*}, \mathbf{0}\right)$. Since each $\nu_{j}$ is linearly independent, it follows that each $v_{j}$ is also linearly independent which serves as a valid basis for the null space of $J\left(p^{*}, \mathbf{0}\right)$. Hence the statement is proved.

The above analysis further leads to the following lemma, which can be easily proved by observing the size of the matrices and the dimensions of the null spaces.

Lemma 4: Suppose at an equilibrium $p^{*}$, the rank of the Jacobian matrix $J_{p^{*}}$ for the singleintegrator system (8) is $k$. Then at the corresponding equilibrium $\left(p^{*}, \mathbf{0}\right)$ the rank of the Jacobian matrix $J\left(p^{*}, \mathbf{0}\right)$ for the double-integrator system (11) is $d n+k$.

We next show a result on an eigenvalue property of $J\left(p^{*}, \mathbf{0}\right)$.

Lemma 5: With the positive definite gain matrix $\mathcal{K}$ as defined previously, the eigenvalues of $J\left(p^{*}, \mathbf{0}\right)$ cannot be purely imaginary.

Proof. Let $\lambda$ be an eigenvalue of $J\left(p^{*}, \mathbf{0}\right)$ with the corresponding eigenvector denoted by $v_{\lambda}=$ $\left[v_{\lambda, p}^{T}, v_{\lambda, v}^{T}\right]^{T}$,

$$
\left[\begin{array}{cc}
\mathbf{0}_{d n \times d n} & I_{d n \times d n} \\
J_{p^{*}} & -\mathcal{K}
\end{array}\right]\left[\begin{array}{l}
v_{\lambda, p} \\
v_{\lambda, v}
\end{array}\right]=\lambda\left[\begin{array}{l}
v_{\lambda, p} \\
v_{\lambda, v}
\end{array}\right]
$$

The above equation can be further rewritten as

$$
\begin{aligned}
v_{\lambda, v} & =\lambda v_{\lambda, p} \\
J_{p^{*}} v_{\lambda, p}-\mathcal{K} v_{\lambda, v} & =\lambda v_{\lambda, v}
\end{aligned}
$$

Multiplying $v_{\lambda, p}^{*}$ (i.e. the complex conjugate transpose of $v_{\lambda, p}$ ) to both sides of the above equation, one can get

$$
\lambda^{2} v_{\lambda, p}^{*} v_{\lambda, p}+\lambda v_{\lambda, p}^{*} \mathcal{K} v_{\lambda, p}-v_{\lambda, p}^{*} J_{p^{*}} v_{\lambda, p}=0
$$

Note that $v_{\lambda, p}$ cannot be a zero vector (otherwise $v_{\lambda}$ will be a zero vector which then contradicts the fact that $v_{\lambda}$ is an eigenvector). Also note that the coefficient $v_{\lambda, p}^{*} \mathcal{K} v_{\lambda, p}$ is positive because $\mathcal{K}$ is positive definite. Hence there cannot exist purely imaginary roots to the above quadratic equation (29), which implies that the eigenvalues of $J\left(p^{*}, \mathbf{0}\right)$ cannot be purely imaginary at any equilibrium.

Remark 4: Note that when $\mathcal{K}=0$ (the damping term is zero) then the above double-integrator system (11) describes a Hamiltonian system. We note one consequence of the above Lemma 5: The non-existence of purely imaginary eigenvalues of the Jacobian $J\left(p^{*}, \mathbf{0}\right)$ implies that Hopf bifurcation in the double-integrator formation system (11) cannot occur.

In the next section, we will interpret these various properties (including the null space properties) of the Hessian and of the Jacobian in the light of convergence of rigid formation systems.

\section{Perspectives from a parameterized double-integrator formation system}

One of the main aims of this paper is to characterize the convergence properties of the doubleintegrator system (11) (and (13)) in terms of those of the reduced-order single-integrator formation system (8). In this section we will consider a family of parameterized formation systems which 
provides a bridge between the single-integrator gradient formation system (8) and the doubleintegrator formation system (11) (and further the flocking control system (13)). Two extreme values of the relevant parameter effectively correspond to single- and double-integrator formations. Intermediate values of the parameter do not correspond to formations, but are relevant to the definition of a sort of homotopy between the two sorts of formation. This parameterization-based idea is inspired by Chiang and Wu (1988), Chiang and Chu (1995) and Dörfler and Bullo (2011), in which similar approaches were employed for the stability and convergence analysis of Hamiltonianlike power systems (Chiang and Wu (1988), Chiang and Chu (1995)) and oscillator networks (Dörfler and Bullo (2011)). A recent paper Oh and Ahn (2014) has briefly discussed this idea in the rigid formation shape control with double-integrator systems. The use of this idea in the context of formation control with shape stabilization and flocking is however novel, which will reveal several important insights on the stability analysis of different types of equilibrium points for double-integrator formation systems. form

Consider the double-integrator formation system with a parameter $\lambda \in[0,1]$ in the following

$$
\mathcal{H}_{\lambda}:\left[\begin{array}{c}
\dot{p} \\
\dot{v}
\end{array}\right]=\underbrace{\left[\begin{array}{cc}
-\lambda I & (1-\lambda) I \\
-(1-\lambda) I & -\mathcal{K}
\end{array}\right]}_{=: S_{\lambda}}\left[\begin{array}{c}
\nabla_{p} \psi \\
\nabla_{v} \psi
\end{array}\right]
$$

In the case that $\lambda=0$, the above system (30) reduces to the double-integrator formation stabilization system shown in (11). In the case that $\lambda=1$, the above system (30) then reduces to the following uncoupled gradient system:

$$
\begin{aligned}
& \dot{p}=-\nabla_{p} \psi \\
& \dot{v}=-\mathcal{K} \nabla_{v} \psi
\end{aligned}
$$

\subsection{Invariance principles}

In the following, we will prove an invariance result which represents a substantial extension of that in Oh and Ahn (2014). This result helps to relate a gradient system (31) and a double-integrator formation stabilization system (11) via the parameterized Hamiltonian system (30).

Theorem 3: For the one-parameter family of dynamical systems $\mathcal{H}_{\lambda}$ in (30), the following statements hold:

- Invariance of equilibrium sets: For all $\lambda \in[0,1]$, the equilibrium set of $\mathcal{H}_{\lambda}$ is given by the set of critical points of the potential function $\psi$ (i.e. the critical points of (31)) and is independent of $\lambda$;

- Invariance of the inertia: For any equilibrium of $\mathcal{H}_{\lambda}$ for all $\lambda \in[0,1]$, the inertia of the Jacobian of the vector function of $\mathcal{H}_{\lambda}$ is equal to that of the Hessian of $\psi$ and is independent of $\lambda$.

Proof. Note that $\mathcal{K}$ is positive definite and is invertible. By the Schur Determinant Formula (Zhang, 2006, Theorem 1.1) it follows from the definition of $S_{\lambda}$ in (30) that $\operatorname{det}\left(S_{\lambda}\right)=\operatorname{det}(-\mathcal{K}) \operatorname{det}(-\lambda I+$ $\left.(1-\lambda)^{2}(-\mathcal{K})^{-1}\right)$, which is non-zero for all $\lambda \in[0,1]$. Hence, the matrix $S_{\lambda}$ is nonsingular for all $\lambda \in[0,1]$, which implies that the equilibrium set of $\mathcal{H}_{\lambda}$ for which the right side of (30) is zero is identical with the equilibrium set for which the right-hand side of (31) is zero, irrespective of the value of $\lambda \in[0,1]$. That is, the equilibrium set of $\mathcal{H}_{\lambda}$ is given by the critical points of $\psi$ : $\mathcal{M}_{\mathcal{H}_{\lambda}}=\left\{\left(p^{*}, v^{*}\right): \nabla_{p} \psi\left(p^{*}\right)=\mathbf{0}, \nabla_{v} \psi\left(v^{*}\right)=\mathbf{0}\right\}$ which is independent of the value of $\lambda$. Thus the first statement is proved. 
The proof of the second statement is inspired by Dörfler and Bullo (2011). Denote the Hessian of $\psi$ as $\nabla_{p, v}^{2} \psi$. The Jacobian of the vector function on the right-hand side of (30) can be written as $J_{\mathcal{H}_{\lambda}}=S_{\lambda} \nabla_{p, v}^{2} \psi$. Denote $A:=-J_{\mathcal{H}_{\lambda}}^{T}, P:=\nabla_{p, v}^{2} \psi$. By noting that $P$ is symmetric, one has

$$
\begin{aligned}
Q: & =\left(A P+P A^{T}\right)=P\left(-S_{\lambda}-S_{\lambda}^{T}\right) P \\
& =P\left[\begin{array}{cc}
2 \lambda I_{d n \times d n} & \mathbf{0}_{d n \times d n} \\
\mathbf{0}_{d n \times d n} & 2 \mathcal{K}
\end{array}\right] P
\end{aligned}
$$

It is obvious that $Q \succeq 0$ for $\lambda \geq 0$, and for $\lambda \neq 0$ we have $\operatorname{null}(Q)=\operatorname{null}(P)$. Hence, by (Carlson and Schneider, 1963, Theorem 5) one can conclude that the non-zero inertia (i.e. the number of $\left.\left\{\nu_{+}, \nu_{-}\right\}\right)$of $A$ is the same as the non-zero inertia of $P$. Also note that $-J_{\mathcal{H}_{\lambda}}$ and $-J_{\mathcal{H}_{\lambda}}^{T}$ have the same set of eigenvalues. These facts further imply that the non-zero inertia of $-J_{\mathcal{H}_{\lambda}}$ is determined by $\nabla_{p, v}^{2} \psi$ and is independent of $\lambda \in(0,1]$. We then consider the case of $\lambda=0$. Note that the eigenspace corresponding to the eigenvalues with zero real parts of $-J_{\mathcal{H}_{\lambda}}$ equals the eigenspace of zero eigenvalues of $\nabla_{p, v}^{2} \psi$ for all $\lambda \in[0,1]$, which implies that the non-zero inertia of $J_{\mathcal{H}_{\lambda}}$ cannot change when $\lambda=0$. In summary, the inertia of $-J_{\mathcal{H}_{\lambda}}$ equals the inertia of $\nabla_{p, v}^{2} \psi$ for all $\lambda \in[0,1]$, and thus the stability property of an equilibrium for (30) is determined by the inertia of the Hessian $\nabla_{p, v}^{2} \psi$ at that equilibrium and is independent of $\lambda$.

Remark 5: The above Theorem 3 can be seen as a generalization of (Oh and Ahn, 2014, Theorem 4.1) (which again is an extension of (Dörfler and Bullo, 2011, Theorem 5.1)). This theorem was presented and used in Oh and Ahn (2014) to show the local asymptotic convergence of a formation stabilization system with double integrators which focused on two special cases: $\lambda=1$ and $\lambda=0$. However, no proof was shown in Oh and Ahn (2014). We note that a proof for the above result is non-trivial, and we also extend the invariance results to the more general case for all $\lambda \in[0,1]$. Furthermore, a by-product of this Theorem 3 shows the equivalence between the eigenspace of the eigenvalues with zero real parts of $J\left(p^{*}, \mathbf{0}\right)$ and the null space of $S_{\lambda} \nabla_{p, v}^{2} \psi$, which generalizes the result in Theorem 2. In the following analysis in Section 4.3, we will show that Theorem 3 gives us additional insights to show the local exponential convergence.

\section{$4.2 \quad$ Relating double-integrator formation systems to single-integrator formation systems}

We now show more results to characterize different equilibrium sets of the double-integrator formation systems, by relating them to the available results in single-integrator formation systems. The following results are direct consequences of Theorem 3.

Corollary 2: Suppose at an equilibrium $p^{*}$ in the equilibrium set $\mathcal{M}_{S}$, the Jacobian matrix $J_{p^{*}}$ for the single-integrator system (8) has $k$ (resp. $j$ ) positive (resp. negative) eigenvalues. Then at the corresponding equilibrium $\left(p^{*}, \mathbf{0}\right)$ in the equilibrium set $\mathcal{M}_{D}$ for the double-integrator system (11), the Jacobian matrix $J\left(p^{*}, \mathbf{0}\right)$ has $k$ (resp. $\left.d n+j\right)$ eigenvalues with positive (resp. negative) real parts.

As shown in Theorem 2, the Jacobian matrix $J\left(p^{*}, \mathbf{0}\right)$ for $(11)$ is always singular at every equilibrium point. Also, the Jacobian matrix $J_{p^{*}}$ (which is the Hessian of the negative potential $V$ ) is always singular, with parts of the null space induced by the rotation and translation invariance of rigid formation shapes.

By assuming that the Hessian is normal hyperbolic (i.e. there is no extra zero eigenvalue for the Hessian other than those induced by shape invariance), Part II of Theorem 3 allows us to employ the inertia information of the Jacobian and of the Hessian to determine the local stability of an equilibrium set. 
Corollary 3: (Property of equilibria in single- and double-integrator formation systems) Suppose the Hessian of the distance potential is normal hyperbolic at a particular equilibrium orbit, and for the single-integrator formation system (8), that particular equilibrium set is stable (resp. unstable). Then for the double-integrator formation system (11), the corresponding equilibrium set is stable (resp. unstable).

Theorem 3 and Corollary 3 imply that available results on stability analysis in single-integrator systems (e.g. Krick et al. (2009), Dörfler and Francis (2010); Park et al. (2014); Sun et al. (2015a)) can be applied here to determine whether an equilibrium set $\mathcal{M}_{D}\left(p^{*}, \mathbf{0}\right)$ is stable or not for the double-integrator formation stabilization system under question. In the following we will show an example.

Since the formation control system is a nonlinear system and it exhibits multiple equilibrium orbits, it is challenging to give a complete picture of the convergence property for different equilibrium sets. The following theorem borrows an existing result from single-integrator formation systems and confirms the instability for a particular set of incorrect equilibria.

In the following analysis, we assume that the target formation is realizable by a nondegenerate configuration in $\mathbb{R}^{d} .{ }^{3}$ Given an equilibrium $p^{*} \in \mathbb{R}^{\text {nd }}$ for the single-integrator formation system (8), we construct a matrix $P^{*}=\left[p_{1}^{*}, p_{2}^{*}, \cdots, p_{n}^{*}\right] \in \mathbb{R}^{d \times n}$ with the $i$-th column being agent $i$ 's position vector $p_{i}^{*}$. We call an equilibrium point $p^{*}$ with $\operatorname{rank}\left(P^{*}\right)<d$ an incorrect degenerate equilibrium for the single-integrator formation system (8), according to the fact that the affine space that embeds $p^{*}$ has dimension less than $d$ and at such degenerate equilibrium the distance potential function $V(p)$ is not zero (i.e. the target shape is not achieved at $p^{*}$ ). Accordingly, one can define the degenerate equilibrium set for the double-integrator formation system (11) as

$$
\tilde{\mathcal{M}}_{D}:=\left\{\left(p^{*}, v^{*}\right): \nabla_{p} V\left(p^{*}\right)=\mathbf{0}, \operatorname{rank}\left(P^{*}\right)<d, v^{*}=\mathbf{0} \in \mathbb{R}^{d n}\right\}
$$

Examples of degenerate equilibria include collinear equilibria (i.e. the equilibrium positions that span a one-dimensional affine space) for formations when agents live in $\mathbb{R}^{2}$, or coplanar equilibria (i.e. the equilibrium positions that span a two-dimensional affine space) for formations when agents live in $\mathbb{R}^{3}$. In the previous literature, properties of degenerate equilibria have been studied for single-integrator formation systems with some special formation shapes. For example, Dörfler and Francis (2010) and Cao, Morse, Yu, Anderson, Dasgupta, et al. (2011) studied in detail the collinear equilibria for a triangular formation target shape, while Park, Jeong, and Ahn (2013) studied the properties of collinear equilibria and coplanar equilibria for a regular tetrahedron target shape. Note under the assumption that the target formation is realizable by a nondegenerate configuration in $\mathbb{R}^{d}$, the set of degenerate equilibria is a subset of the set of incorrect equilibria at which the formation has an incorrect shape.

According to the above invariance principle, one can obtain the following stability result.

Theorem 4: (Instability of degenerate equilibrium) Suppose the target formation is realizable by a nondegenerate configuration in $\mathbb{R}^{d}$. A degenerate equilibrium set $\tilde{\mathcal{M}}_{D}$ for the double-integrator formation stabilization system (11) is unstable.

Proof. In Sun et al. (2015a), it has been proved that any degenerate equilibrium $p^{*}$ is unstable for the single-integrator formation system (8). As a consequence of Theorem 3, the degenerate equilibrium set $\tilde{\mathcal{M}}_{D}$ characterized by the additional condition $\operatorname{rank}\left(P^{*}\right)<d$ is unstable for the corresponding double-integrator formation stabilization system (11).

Remark 6: Note that in Theorem (4) we do not need to assume that the Hessian of $-V$ (and the associated Jacobian $\left.J\left(p^{*}, \mathbf{0}\right)\right)$ are normal hyperbolic. This is because the existence of at least one

${ }^{3}$ For example, a target triangle formation shape with three given distances satisfying triangle inequality can be realized in $\mathbb{R}^{2}$
but not in $\mathbb{R}^{1}$. In this case, any collinear equilibrium that only spans $d=1$-dimensional affine space is a degenerate equilibrium. 


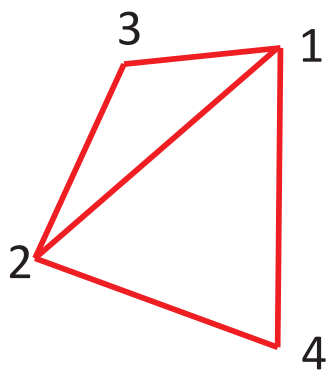

(a)

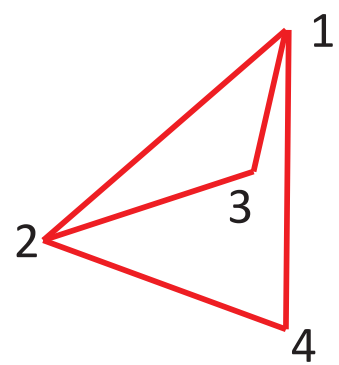

(b)

Figure 1. An example of a pair of flip/flex formations. (a) A target formation defined by 5 inter-agent distances. (b) A flip version of the target formation with the same set of 5 desired distances. In rigid formation control, they are both considered as correct equilibria for a target formation specified by given desired distances.

negative eigenvalue ensures the existence of unstable manifold, which can be uniquely determined and is tangent to the unstable subspaces corresponding to the negative eigenvalue (c.f. the Nonhyperbolic Hartman-Grobman Theorem (Meiss, 2007, Page 189, Theorem 5.9)). As proved in Sun et al. (2015a), unstable degenerate equilibria for single-integrator formation systems are actually either local maximum or saddle points, and for saddle points there exist initial positions (the set of which has measure zero) forming an affine space of lower dimensions such that the single-integrator formation system cannot escape from that lower dimensional space and will converge to one of such degenerate equilibria. We will verify in simulations that, for double-integrator formation systems, even if agents start with initial positions in a lower dimensional space, the formation system can still converge to a correct formation shape instead of a degenerate equilibrium with an incorrect shape.

Remark 7: One needs to distinguish the set of degenerate equilibria and the set of flip/flex formations. According to the definition of degenerate equilibrium in (33), the set of such equilibrium points is defined by the requirement that the agent positions at such an equilibrium point span an affine space of less than full dimension of its containing space. However, flip/flex formations refer to different realizations (up to translation, rotation and reflection) of a target formation with a given set of distances (see Remark 3). Thus, these two concepts are not related. We provide two examples in Fig. 1 and Fig. 2 to illustrate these two concepts. Actually, in the distance-based formation setup, a target formation is defined by a set of inter-agent distances, which do not distinguish flip/flex formations. This is because that the sets of edges and corresponding distance constraints do not uniquely define the relative positions of the agents. In other words, the distance constraints do not specify the formation shape up to a rotation, translation, or reflection Anderson et al. (2008); Krick et al. (2009). In this paper, since we only focus on local convergence of a gradient-type system, flip/flex ambiguity can be avoided by assuming that the initial formation is closer to the desired shape than the flip/flex version which guarantees that the formation will converge to the desired shape instead of a flip/flex formation due to the gradient-descent property (see relevant discussions in Krick et al. (2009)). If one wants to always avoid flip/flex ambiguities, then the target formation should be globally rigid, or should be defined in a different way by including constraints on flip/flex formations. Consequently, the corresponding formation controllers should also include additional terms involving the avoidance of flip/flex ambiguity.

Remark 8: In many published papers on rigid formation control (see e.g. Deghat et al. (2015); Dörfler and Francis (2010); Krick et al. (2009); Oh et al. (2015); Sun et al. (2015a)), it is a standard assumption that the target formation is infinitesimally rigid, and during the convergence process the instantaneous formation shape at an arbitrary instant of time is ensured also to be 


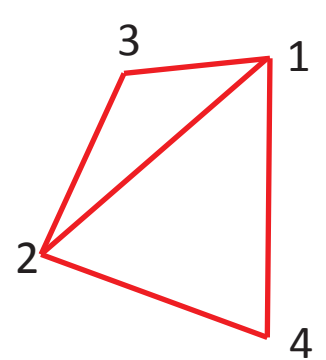

(a)

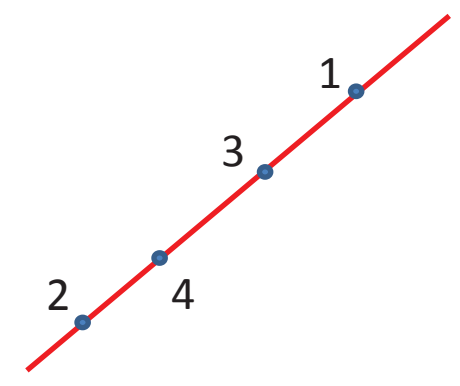

(b)

Figure 2. An example on a correct equilibrium and a degenerate equilibrium for a 4-agent formation shape with 5 given distances. (a) The correct formation shape, in which all the 5 distances are achieved. (b) An example of collinear degenerate equilibrium in $\mathbb{R}^{2}$, in which all the 4 agents converge to a line formation. In Sun et al. (2015a), it has been proved that degenerate equilibria always exist, and if all agents are initially placed at collinear positions then the single-integrator rigid formation system will converge to one of such collinear degenerate equilibria under the control (8).

infinitesimally rigid (i.e., the rigidity matrix always attains its maximum rank as in (4) at any time during the convergence process). This cannot be achieved for arbitrary initial conditions, and for this reason most papers only consider local convergence, that is, the initial formation is chosen to be infinitesimally rigid and close to the target formation. Under this assumption, the infinitesimal rigidity of the instantaneous formation shape during the convergence can be guaranteed due to the gradient property of the dynamical system (which ensures that the distance error vector is nonincreasing) and the continuity dependence of the rigidity matrix to agents' positions. In Section 4.3 we will also use this assumption to ensure local exponential convergence. The rank-preserving property for single-integrator rigid formation control systems, established in Sun et al. (2015a), is however a different concept, which in most cases is not related to the rank of the rigidity matrix $R$. This can be seen from the definition of degenerate equilibria in (33) with the rank condition of the matrix $P$. The rank of $P$ is the dimension of the ambient affine space containing all agents, and is not the same to the rank of $R$ (or the infinitesimal rigidity as shown in (4)). We have tried to apply the rank-preserving theory (see (Helmke and Moore, 2012, Chapter 5)) to determine whether the global preservation of the infinitesimal rigidity holds during the convergence under the gradient flow (8), but cannot obtain the result. This is because one cannot transform the matrix differential system involving $R$ into the standard form of a rank-preserving matrix differential equation shown in (Helmke and Moore, 2012, Lemma 1.22, Chapter 5). There are some results on the global preservation of infinitesimal rigidity during the convergence for special formations (e.g. triangular formation Dörfler and Francis (2010), or tetrahedral formation Park et al. (2014)), since in these special cases the rank of $P$ can be determined from the rank of $R$. Whether the infinitesimal rigidity can be preserved for general formations in a global sense beyond the local convergence from arbitrary initial formations (that are infinitesimally rigid) is still an open problem.

The following result further clarifies the topological conjugacy ${ }^{4}$ of the two trajectories from (11) and (31), which indicates that they can be continuously deformed to match each other while preserving the parameterization of time.

Theorem 5: (Topological conjugacy) Consider the double-integrator formation system (11) and the uncoupled single-integrator formation gradient system (31) modelled by the same undirected and rigid underlying graph and the same target formation. Then locally near a correct equilibrium set, their trajectories are topologically conjugate.

${ }^{4}$ Two flows $\varphi_{t}: A \rightarrow A$ and $\psi_{t}: B \rightarrow B$ are conjugate if there exists a homeomorphism $h: A \rightarrow B$ such that for each $x \in A$ and $t \in \mathbb{R}$, there holds $h\left(\varphi_{t}(x)\right)=\psi_{t}(h(x))$. For more discussions on topological conjugacy, the readers are referred to (Meiss, 2007, Chapter 4.7). 
Proof. The proof is inspired by (Dörfler and Bullo, 2011, Theorem 5.3). As discussed above, any correct equilibrium in the set of correct equilibria $\left(p^{*}, \mathbf{0}\right)$ for $(11)$ is not isolated but forms an equilibrium manifold with dimension $d(d+1) / 2$, which is induced by translational and rotational invariance. However, by factoring out the translational and rotational invariance, the correct equilibrium corresponds to an isolated equilibrium in a quotient space. Such quotient space for the linearized system of (11) can be identified with the orthogonal complement of the null space induced by shape invariance, and the Jacobian matrix $J\left(p^{*}, \mathbf{0}\right)$ is normal hyperbolic (i.e. hyperbolic in that quotient space). According to the Hartman-Grobman theorem (Meiss, 2007, Theorem 4.13), the trajectories of the vector fields (11) and (31) are locally topologically conjugate to the flow generated by their respective linearized vector fields near the correct equilibrium. Also as proved in Theorem 3, the linearized Jacobian matrix for each flow (11) and (31) has the same hyperbolic inertia (besides the same common center eigenspace of the same dimension induced by shape invariance). According to (Meiss, 2007, Theorem 4.12), this indicates that the corresponding linearized dynamics are topologically conjugate. In summary, the trajectories generated by the vector fields (11) and (31) are locally topologically conjugate near the correct equilibrium set.

We note that such topological conjugacy property has been observed in several other practical systems modelled by double integrators, including power systems Chiang and Chu (1995) and oscillator synchronization Dörfler and Bullo (2011). Here we show that it also holds for rigid formation control systems.

\subsection{Exponential convergence of double-integrator formation systems}

The following analysis in particular shows the convergence to a point in a correct equilibrium set and its convergence rate in the rigid formation control problem under discussion. With a slight abuse of terminology, we will call the matrix $J_{p^{*}}$ normal negative definite if its null space only consists of the subspace induced by shape invariance and all non-zero eigenvalues are positive.

Lemma 6: (Local exponential convergence) The trajectory of (11) converges locally exponentially fast to an equilibrium point $\left(p^{*}, \mathbf{0}\right)$ in an equilibrium set, if and only if $J_{p^{*}}$ is normal negative definite at $p^{*}$ in the vector space orthogonal to the null space induced by shape invariance.

The proof for Lemma 6 is omitted but can be easily inferred from Theorem 3 as well as Corollary 2 and Corollary 3. By the assumption that the target formation is infinitesimally rigid, the above Lemma 6 indicates that the correct equilibrium is exponentially stable. The following gives a more detailed explanation on this exponential convergence statement.

Let us take 2-D formation systems as an example to interpret the above Lemma 6 and to determine the exponential convergence of double-integrator formation systems. From the characterization of the null space of the Jacobian matrix, the inertia of the Jacobian matrix at a point in a particular equilibrium orbit is independent of the choice of that point. If at an equilibrium point $p^{*}$ in a particular equilibrium set, the Jacobian $J_{p^{*}}$ of a 2-D single-integrator system (8) has inertia as $\left\{\nu_{+}, \nu_{-}, \nu_{0}\right\}=\{0,2 n-3,3\}$, then such equilibrium set is locally exponentially stable for (8). Actually, by invoking the Center Manifold Theorem Carr (1981) and by assuming that the target formation is infinitesimally rigid, it has been proven in Krick et al. (2009) that the position of each agent in the gradient system $\dot{p}=-\nabla_{p} \psi$ in (31) converges locally exponentially fast to an equilibrium point in the correct equilibrium set, with the equilibrium point dependent on the initial condition. ${ }^{5}$

By combining this result with the statement in Lemma 6, one can show that if at an equilibrium point $\left(p^{*}, \mathbf{0}\right)$ in a particular equilibrium set $\mathcal{M}_{D}\left(p^{*}, \mathbf{0}\right)$, the Jacobian $J\left(p^{*}, \mathbf{0}\right)$ of a 2-D double-

\footnotetext{
${ }^{5}$ Another way for proving the exponential convergence is to focus on the relative position dynamics or distance error dynamics; see e.g. Dörfler and Francis (2010); Sun et al. (2016).
} 
integrator system (11) has inertia as $\left\{\nu_{+}, \nu_{-}, \nu_{0}\right\}=\{0,4 n-3,3\}$, then such an equilibrium set is locally exponentially stable and the trajectory of (11) converges locally exponentially fast to an equilibrium point in this equilibrium set. In particular, if the target formation is infinitesimally rigid, then the double-integrator formation system (11) converges locally exponentially fast to an equilibrium point in the correct equilibrium set with desired formation shape.

Furthermore, according to the definition of the distance error vector $e$ in (5), we can conclude from the above discussion that inter-agent distances also converge locally exponentially fast to the desired values. It is worth mentioning that there exist other ways for proving local convergence for double-integrator formation systems (see e.g. Oh and Ahn (2014) by using the Lojasiewicz's inequality for a gradient flow, Jiang, Deghat, and Anderson (2016) with the usage of the Malkin theorem, and Dimarogonas and Johansson (2008), Sun et al. (2015b), Zhang et al. (2015) by invoking Barbalat's Lemma and Lyapunov argument), all without however showing how fast the convergence is. Note that the exponential convergence delivered by our arguments is a crucial property for studying robustness issues in rigid formation control systems Mou, Morse, Belabbas, Sun, and Anderson (2016). We mention that the key idea of Theorem 3 and Lemma 6 in this section has been employed in a recent paper Garcia de Marina, Jayawardhana, and Cao (2016) to study a double-integrator formation stabilization system with constant distance mismatch. In our future research, we will show that the exponential convergence established in this paper is a key factor in explaining the robustness property of double-integrator rigid formation system and flocking system in the presence of more general perturbations.

\section{Extensions to double-integrator flocking systems}

At the beginning of Section 3 we have established explicit forms and some properties of equilibrium points for double-integrator flocking systems (13). In this section we will provide brief discussions to show that the obtained results in previous sections on double-integrator formation stabilization systems (11) can be extended to formation flocking systems (13). Note that in the formation flocking system, the motion of the whole formation can be broken into a combination of two motions Deghat et al. (2015), i.e., that of the centroid, and that of the formation relative to the centroid. The latter motion is like that already analyzed in the previous sections. The former motion (that of the centroid) is simple to analyze. We now show how this works.

Recalling the notations in Section 3.1, we have used $p_{c}$ to denote the formation centroid, and $v_{c}$ to denote the average velocity of all the agents, i.e., $v_{c}(t)=\dot{p}_{c}=\frac{1}{n} \sum_{i=1}^{n} v_{i}(t)$. Observe that $v_{c}(t)$ is time-invariant as shown in Lemma 2 . We then define the relative position vector to the formation centroid as $\bar{p}_{i}=p_{i}-p_{c}$, and the relative velocity vector $\bar{v}_{i}=\dot{\bar{p}}_{i}=\dot{p}_{i}-\dot{p}_{c}$. Note that there holds $\bar{p}_{i}-\bar{p}_{j}=p_{i}-p_{j}$ and $\bar{v}_{i}-\bar{v}_{j}=v_{i}-v_{j}$. Also note there holds $\dot{\bar{v}}_{i}=\ddot{\bar{p}}_{i}$ since $v_{c}$ is constant. Hence, one can further transform (13) into the following equivalent system by factoring out the formation centroid dynamics:

$$
\begin{aligned}
\dot{\bar{p}}_{i} & =\bar{v}_{i} \\
\dot{\bar{v}}_{i} & =\sum_{j \in \mathcal{N}_{i}}\left(\bar{v}_{j}-\bar{v}_{i}\right)+\sum_{j \in \mathcal{N}_{i}} e_{k_{i j}}\left(\bar{p}_{j}-\bar{p}_{i}\right)
\end{aligned}
$$

By introducing the composite relative position vector $\bar{p}=\left[\bar{p}_{1}, \bar{p}_{2}, \cdots, \bar{p}_{n}\right]^{T}$ and the velocity disagreement vector as $\bar{v}=\left[\bar{v}_{1}, \bar{v}_{2}, \cdots, \bar{v}_{n}\right]^{T}$, one can rewrite the above system (34) in a compact form

$$
\begin{aligned}
& \dot{\bar{p}}=\bar{v} \\
& \dot{\bar{v}}=-\mathcal{L} \bar{v}-R^{T}(\bar{p}) e(\bar{p})
\end{aligned}
$$



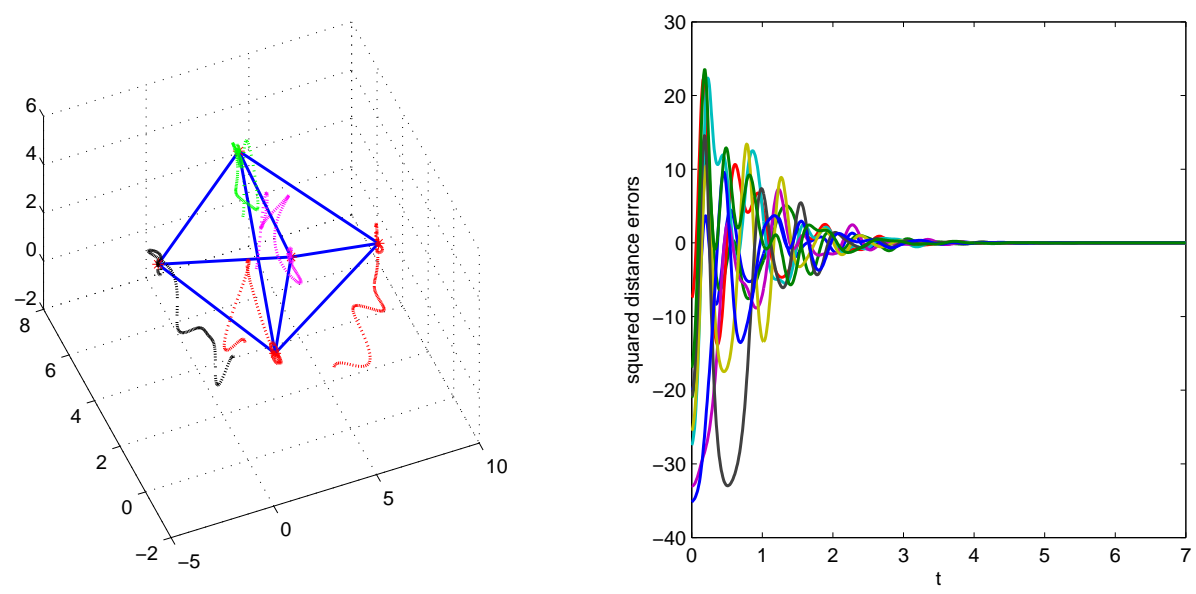

Figure 3. Simulation on shape stabilization control of a double tetrahedron formation in the 3-D space with double-integrator systems.

Since $\operatorname{null}(L)=\operatorname{span}\left\{\mathbf{1}_{n}\right\}$, there holds $\operatorname{null}(\mathcal{L})=\operatorname{span}\left\{\mathbf{1}_{n} \otimes I_{d \times d}\right\}$. Thus $\mathcal{L}$ is positive definite by restricting it to the vector space of $\operatorname{span}\left(\mathbf{1}_{n} \otimes I_{d \times d}\right)^{\perp}$ (where the superscript ${ }^{\perp}$ denotes orthogonal complement) in which the velocity disagreement vector $\bar{v}$ lives. From this viewpoint, $\mathcal{L}$ serves the same role as the positive definite gain matrix $\mathcal{K}$ in (11). Note that the equilibrium set $\mathcal{M}_{F}$ of (13) can be restated in the new coordinates as $\overline{\mathcal{M}}_{F,(35)}=\left\{\left(\bar{p}^{*}, \bar{v}^{*}\right): \nabla_{\bar{p}} V\left(\bar{p}^{*}\right)=\mathbf{0}, \bar{v}^{*}=\mathbf{0}\right\}$ for the transformed system (35). Thus, the transformed system (35) has the same structure as the double-integrator formation system (11), and all previous results on the equilibrium properties and parameterized system analysis for the double-integrator formation system (11) can be readily extended to (35).

\section{Illustrative examples on convergence}

In the simulations we consider formation control examples in the 3-D space. The target formation shape is supposed to be a double tetrahedron formation with 9 edges, with the desired distances for each edge being 6 . The initial positions and initial velocities for each agent are chosen randomly, but we also ensure that the initial formation shape is close to the target one. The formation stabilization result is shown in Figure 3, which illustrates the trajectories of each agent, together with the initial shape and final shape, and the trajectories of each distance error. The formation flocking result is shown in Figure 4, which illustrates the trajectories of each agent, the flocking behavior, and the trajectories of each distance error. It can also be seen from simulations that the convergence of each distance error vector in both cases is exponentially fast.

Note that the above examples only show local convergence to the target formation shape. The following simulation shows a comparison of convergence results between single-integrator formation models and double-integrator formation models. We assume the same target formation shape as described above, and choose the initial positions for all agents as $p_{1}(0)=[4,4,0]^{T}$, $p_{2}(0)=[0,0,0]^{T}, p_{3}(0)=[4,0,0]^{T}, p_{4}(0)=[5,0,0]^{T}$ and $p_{5}(0)=[-4,-10,0]^{T}$. The simulation result with single-integrator model is depicted in Figure 5, which shows the convergence to an incorrect formation shape in a degenerate 2-D plane when agents' initial positions are chosen in that plane (which has measure zero in the whole space $\mathbb{R}^{3}$ ). The converged equilibrium in this case is $p_{1}^{*}=[3.2992,3.8112,0]^{T}, p_{2}^{*}=[-1.5078,0.2206,0]^{T}, p_{3}^{*}=[4.0052,-2.1471,0]^{T}$, $p_{4}^{*}=[4.0052,-2.1471,0]^{T}$ and $p_{5}^{*}=[-0.8018,-5.7376,0]^{T}$, which span the same plane as the initial positions. The convergence to an incorrect equilibrium (more specifically, a degenerate 

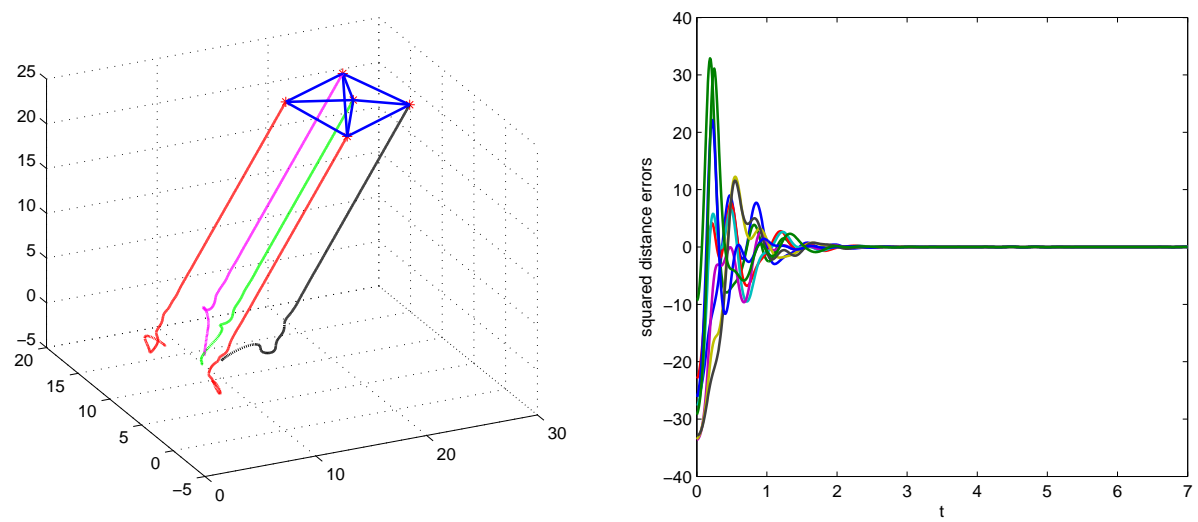

Figure 4. Simulation on formation flocking control of a double tetrahedron formation in the 3-D space with double-integrator systems.
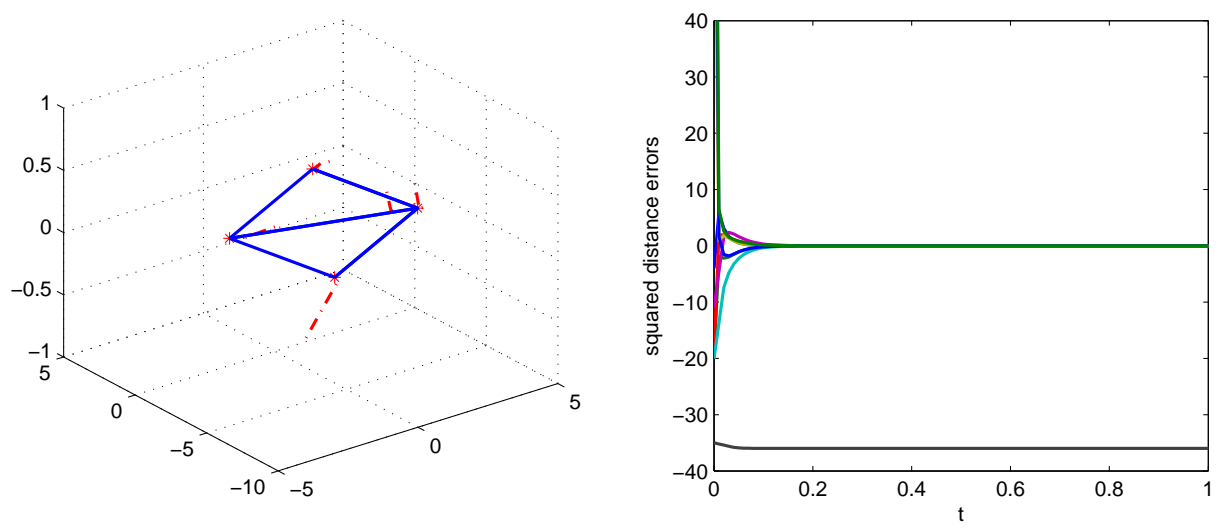

Figure 5. Simulation on formation shape control of a double tetrahedron formation in the 3-D space with single-integrator systems. The initial conditions are chosen to be in a plane in the 3-D space, and the formation converges to an incorrect formation shape that lives in that plane (i.e. an incorrect planar equilibrium formation).

incorrect equilibrium) is due to the rank-preserving property of single-integrator rigid formation systems as discussed in Sun et al. (2015a).

We then use the same initial positions (and random initial velocities) to perform the simulation with double-integrator models, and observe the convergence to the correct formation shape as shown in Figure 6. Actually, as proved in Sun et al. (2015a), the incorrect equilibrium formation illustrated in Figure 5 is a saddle point. Such an equilibrium point is unstable for both singleintegrator formation systems and double-integrator formation systems (see Theorem 4). However, this simulation also shows one advantage of using double-integrator formation models against single-integrator formation models. For double-integrator formation systems, even if one chooses initial positions that live in a lower-dimensional space, the formation system will not always live in that degenerate space. That is, double-integrator formation systems can escape from degenerate positions (e.g. collinear positions or coplanar positions) and thus will avoid the convergence to an incorrect degenerate equilibrium even if agents' positions are initially placed in a lower dimensional space. This is due to that the rank-preserving property does not hold for rigid formation systems modelled by double-integrator dynamics (for details, see Sun et al. (2015a)). 

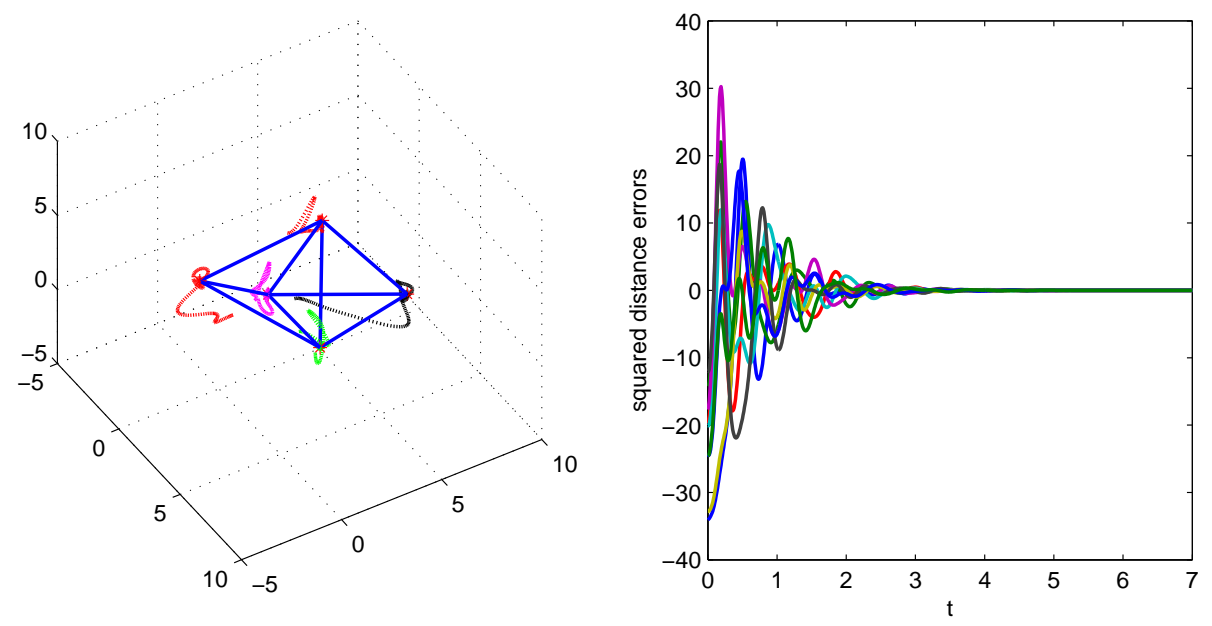

Figure 6. Simulation on formation shape control of a double tetrahedron formation in the 3-D space with double-integrator systems. The formation converges to a correct formation shape, even one chooses degenerate coplanar initial positions.

\section{Conclusions}

In this paper we have considered formation control systems modelled by double integrators, which include the formation stabilization model and flocking control model. Due to the multi-equilibrium property caused by the nonlinear rigid formation controller, a complete analysis of the convergence is quite challenging. This paper serves as a further step to understand the dynamical behavior of such formation control systems. Novel properties of the system dynamics and convergence analysis for different equilibria are discussed by analyzing certain properties of the linearized systems and a parameterized Hamiltonian-like system. Some key results are summarized as follows:

- We discuss the measurement requirement for individual agents and establish the independence of a global coordinate frame for implementing the formation controller.

- Certain properties of the Jacobian matrix for double-integrator formation systems (e.g. the rank, null spaces and eigenvalues) are characterized.

- Invariance principles concerning the equilibrium set and local stability are established for a family of parameterized Hamiltonian-like system, which builds the link between singleintegrator formation systems and double-integrator formation systems. Thus, available results on equilibrium analysis in single-integrator rigid formation systems reported in the vast literature can be extended to double-integrator formation systems.

- Several criteria for determining the stability and convergence of equilibrium sets for doubleintegrator formation systems are proposed.

Future research will focus on the robustness property for rigid formation systems modelled by double integrators. In Mou et al. (2016), it has been shown that in the case of single-integrator rigid formation system, undesired rigid motions will occur if there exist inconsistent distances perceived by neighboring agent pairs. It will be interesting to investigate whether such rigid motions still occur in double-integrator formation systems with inconsistent distances or distance measurement errors. The main tools in Mou et al. (2016) include linearization analysis and exponential stability of the single-integrator system. Thus, we expect that the linearization technique and Jacobian matrix analysis in Section 3, as well as the parameterized system analysis and the exponential convergence established in Section 4 of this paper, will be fundamental results to establish the robustness property for double-integrator rigid formation systems. 


\section{Acknowledgements}

This work is supported by NICTA, which is funded by the Australian Government as represented by the Department of Broadband, Communications and the Digital Economy and the Australian Research Council through the ICT Centre of Excellence program. It is also partially supported by National Natural Science Foundation of China No. 61501282. B. D. O. Anderson was supported by the ARC under grant DP130103610. H.-S. Ahn is supported by the National Research Foundation of Korea under Grant NRF-2013R1A2A2A01067449. Z. Sun is supported by the Prime Minister's Australia Asia Incoming Endeavour Postgraduate Award from Australian Government.

\section{References}

Anderson, B. D. O., Helmke, U., 2014. Counting critical formations on a line. SIAM Journal on Control and Optimization 52 (1), 219-242.

Anderson, B. D. O., Yu, C., Fidan, B., Hendrickx, J. M., 2008. Rigid graph control architectures for autonomous formations. Control Systems Magazine, IEEE 28 (6), 48-63.

Cai, X., De Queiroz, M., 2014. Multi-agent formation maneuvering and target interception with double-integrator model. In: Proc. of the American Control Conference. IEEE, pp. 287-292.

Cao, M., Morse, A. S., Yu, C., Anderson, B. D. O., Dasgupta, S., et al., 2011. Maintaining a directed, triangular formation of mobile autonomous agents. Communications in Information and Systems 11 (1), 1.

Cao, Y., Yu, W., Ren, W., Chen, G., 2013. An overview of recent progress in the study of distributed multi-agent coordination. Industrial Informatics, IEEE Transactions on 9 (1), 427-438.

Carlson, D., Schneider, H., 1963. Inertia theorems for matrices: The semidefinite case. Journal of Mathematical Analysis and Applications 6 (3), 430-446.

Carr, J., 1981. Applications of centre manifold theory. Springer.

Chen, X., Belabbas, M.-A., Başar, T., 2015. Formation control with triangulated laman graphs. arXiv preprint arXiv:1412.6958.

Chiang, H.-D., Chu, C.-C., 1995. Theoretical foundation of the bcu method for direct stability analysis of network-reduction power system models with small transfer conductances. Circuits and Systems I: Fundamental Theory and Applications, IEEE Transactions on 42 (5), 252-265.

Chiang, H.-D., Wu, F. F., 1988. Stability of nonlinear systems described by a second-order vector differential equation. Circuits and Systems, IEEE Transactions on 35 (6), 703-711.

Deghat, M., Anderson, B. D. O., Lin, Z., 2015. Combined flocking and distance-based shape control of multi-agent formations. Automatic Control, IEEE Transactions on, in press, DOI: 10.1109/TAC.2015.2480217.

Dimarogonas, D. V., Johansson, K. H., 2008. On the stability of distance-based formation control. In: Proc. of the 47th Conference on Decision and Control. IEEE, pp. 1200-1205.

Dörfler, F., Bullo, F., 2011. On the critical coupling for kuramoto oscillators. SIAM Journal on Applied Dynamical Systems 10 (3), 1070-1099.

Dörfler, F., Francis, B., 2010. Geometric analysis of the formation problem for autonomous robots. Automatic Control, IEEE Transactions on 55 (10), 2379-2384.

Garcia de Marina, H., Jayawardhana, B., Cao, M., 2016. Taming inter-distance mismatches in formation-motion control for rigid formations of second-order agents. IEEE Transactions on Automatic Control, submitted. Availabe at Arxiv: http://arxiv.org/abs/1604.02943.

Hahn, W., Baartz, A. P., 1967. Stability of motion. Vol. 138. Springer.

Helmke, U., Anderson, B. D. O., 2013. Equivariant morse theory and formation control. In: Proc. of the 51st Annual Allerton Conference on Communication, Control, and Computing. IEEE, pp. $1576-1583$.

Helmke, U., Moore, J. B., 2012. Optimization and dynamical systems. Springer Science \& Business 
Media.

Hendrickson, B., 1992. Conditions for unique graph realizations. SIAM Journal on Computing $21(1), 65-84$.

Jiang, B., Deghat, M., Anderson, B. D. O., 2016. Velocity consensus and formation shape control using distance-only measurements. IEEE Transactions on Automatic Control, accepted. Available at Arxiv: http://arxiv.org/abs/1411.3841.

Krick, L., Broucke, M. E., Francis, B. A., 2009. Stabilisation of infinitesimally rigid formations of multi-robot networks. International Journal of Control 82 (3), 423-439.

Meiss, J. D., 2007. Differential dynamical systems. Vol. 14. SIAM.

Meng, Z., Anderson, B. D. O., Hirche, S., 2016. Formation control with mismatched compasses. Automatica 69, 232-241.

Mou, S., Morse, A. S., Belabbas, M. A., Sun, Z., Anderson, B. D. O., 2016. Undirected rigid formations are problematic. Automatic Control, IEEE Transactions on, in press, DOI: 10.1109/TAC.2015.2504479.

Oh, K.-K., Ahn, H.-S., 2014. Distance-based undirected formations of single-integrator and doubleintegrator modeled agents in n-dimensional space. International Journal of Robust and Nonlinear Control 24 (12), 1809-1820.

Oh, K.-K., Park, M.-C., Ahn, H.-S., 2015. A survey of multi-agent formation control. Automatica $53,424-440$.

Olfati-Saber, R., 2006. Flocking for multi-agent dynamic systems: Algorithms and theory. Automatic Control, IEEE Transactions on 51 (3), 401-420.

Park, M.-C., Jeong, K., Ahn, H.-S., 2013. Control of undirected four-agent formations in 3dimensional space. In: Proc. of the 52nd Conference on Decision and Control. IEEE, pp. 14611465.

Park, M.-C., Sun, Z., Anderson, B. D. O., Ahn, H.-S., 2014. Stability analysis on four agent tetrahedral formations. In: Proc. of the 53rd IEEE Conference on Decision and Control. pp. 631-636.

Qin, J., Yu, C., 2013. Coordination of multiagents interacting under independent position and velocity topologies. Neural Networks and Learning Systems, IEEE Transactions on 24 (10), $1588-1597$.

Ramazani, S., Selmic, R. R., de Queiroz, M., 2015. Stabilization of non-planar multi-agent layered formations with double integrator model. In: IEEE Conference on Control Applications. IEEE, pp. $1386-1391$.

Ren, W., 2008. On consensus algorithms for double-integrator dynamics. Automatic Control, IEEE Transactions on 53 (6), 1503-1509.

Rozenheck, O., Zhao, S., Zelazo, D., 2015. A proportional-integral controller for distance-based formation tracking. In: Proc. of the 2015 European Control Conference. pp. 1687-1692.

Sun, Z., Anderson, B. D. O., 2015. Rigid formation control systems modelled by double integrators: system dynamics and convergence analysis. In: Proc. of the 2015 Australian Control Conference. pp. 241-246.

Sun, Z., Helmke, U., Anderson, B. D. O., 2015a. Rigid formation shape control in general dimensions: an invariance principle and open problems. In: Proc. of the 54th IEEE Conference on Decision and Control. pp. $6095-6100$.

Sun, Z., Mou, S., Anderson, B. D. O., Cao, M., 2016. Exponential stability for formation control systems with generalized controllers: a unified approach. Systems and Control Letters 93, 50-57.

Sun, Z., Mou, S., Deghat, M., Anderson, B. D. O., 2015b. Finite time distributed distanceconstrained shape stabilization and flocking control for d-dimensional undirected rigid formations. International Journal of Robust and Nonlinear Control, in press, DOI: 10.1002/rnc.3477.

Tanner, H. G., Jadbabaie, A., Pappas, G. J., 2007. Flocking in fixed and switching networks. Automatic Control, IEEE Transactions on 52 (5), 863-868.

Tian, Y.-P., Wang, Q., 2013. Global stabilization of rigid formations in the plane. Automatica 
49 (5), 1436-1441.

Vasile, C.-I., Schwager, M., Belta, C., 2015. SE(N) Invariance in Networked Systems. In: Proc. of the 2015 European Control Conference. IEEE, pp. 186-191.

Wiggins, S., 2003. Introduction to applied nonlinear dynamical systems and chaos. Vol. 2. Springer Science \& Business Media.

Zhang, F. (Ed.), 2006. The Schur complement and its applications. Vol. 4. Springer Science \& Business Media.

Zhang, P., de Queiroz, M., Cai, X., 2015. Three-dimensional dynamic formation control of multiagent systems using rigid graphs. Journal of Dynamic Systems, Measurement, and Control 137 (11), 111006. 